\title{
Associação congenérica de Ceriodaphnia cornuta Sars 1886 e Ceriodlaphnia reticulata (Jurine) 1820 (Crustacea, Cladocera) na Represa de Curuá-Una, Pará, Brasil (*)
}

\author{
Barbara A. Robertson (**)
}

\section{Resumo}

Um estudo conduzido na Represa de Curuá-Una, Pará, mostra evidências para três mecanismos de segregação (separaçẫo no tempo, no espaço, e uma diferença em tamanho suficiente para implicar em hábitos alimentares diferentes) atuaram em conjunto para permitir a coexistência das espécies congenéricas Ceriodaphnia cornuta e Ceriodaphnia reticulata.

\section{INTRONUÇÃo}

De acordo com Pennak (1957), em uma amostra de plâncton limnético, é raro um gênero de Copepoda ou Cladocera ser representado por mais de uma espécie. O mesmo autor argumenta que a presença de duas ou mais espécies de um mesmo gênero de crustáceo planctônico é uma situaçăo difícil de conciliar com a hipótese de Gause, dado que espécies congenéricas provaveimente competem por nichos muito semelhantes. Contudo, quando isto ocorre, Pennak (1957) observa que as espécies apresentam periodicidades dessemelhantes, o que resulta nos máximos das populaçōes ocorrerem em épocas diferentes, e/ou uma segregaçäo vertical na coluna d'água, e/ou tamanhos suficientemente diferentes para implicarem em hábitos alimentares também diferentes. Estes três mecanismos de segregação, enquanto bem documentados em copépodos (Pennak, 1957; Hutchinson, 1967; Sandercock. 1967; Hardy, 1980; Bowers, 1980), têm sido relativamente pouco estudados em cladóceros.

Durante um estudo da composição, abundância e distribuição de Cladócera realizado na Represa de Curuá-Una (Robertson, 1980), a presença e dominância numérica de Ceriodaphnia cornuta e $C$. reticulata em uma das es- tações de coleta providenciou uma oportunidade para estudar um exemplo interessante de associações congenéricas em cladóceros. Neste tarbalho, são estudados a variação, no tempo e no espaço, na densidade dos animais, e a diferença nos tamanhos de $C$. cornuta e $C$. reticulata.

\section{DESCRIÇÃO DA ÁREA DE ESTUdO}

A Usina Hidrelétrica de Curuá-Una situa-se a $02^{\circ} 48^{\prime} 38^{\prime \prime}$ ' $S$ e $54^{\circ} 18^{\prime} 55^{\prime \prime}$ W no Município de Santarém, Estado do Pará. (Fig. 1) .

A Represa recebe água do rio Curuá-Una e dos seus afluentes, o rio Moju e o rio Mujuí

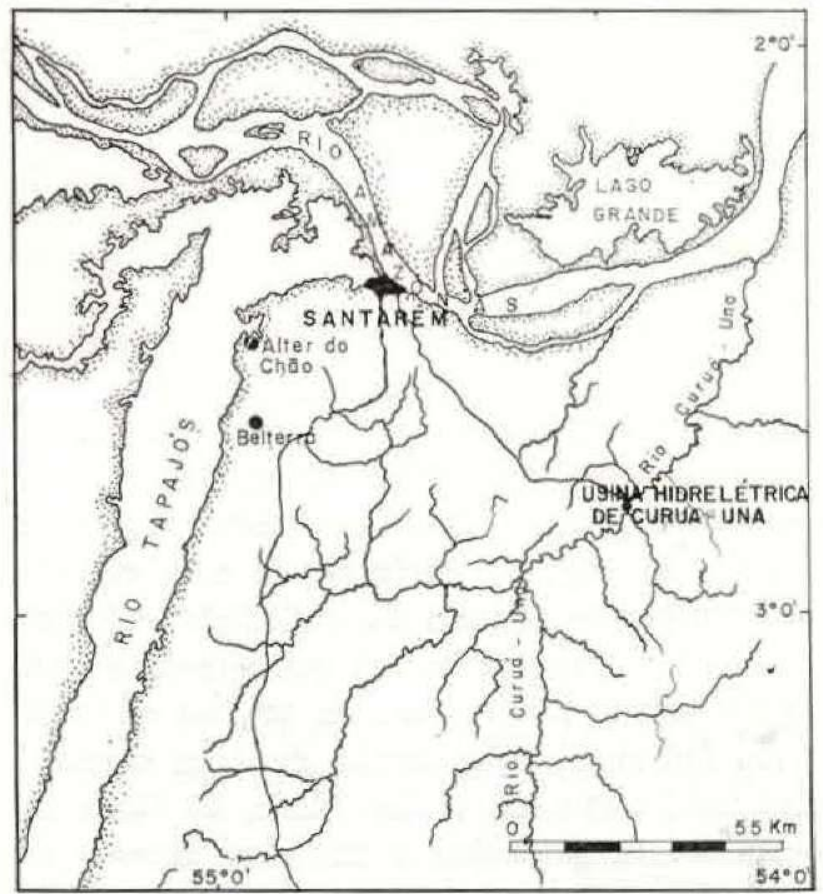

Fig. 1 - Mapa de localização da Usina Hidrelétrica de Curuá-Una (Projeto Radam, 1976).

(") - Parte da tese de Mestrado apresentada ao curso de Pós-graduação da FUA-INPA/CNPq, em 1980. Projeto financiado pelo Projeto Curuá-Una, Convênio CNPq/INPA-ELETRONORTE.

(") - Instituto Nacional de Pesquisas da Amazônia, Manaus. 
dos Campos. Na cota de $68,0 \mathrm{~m}$, o reservatório cobre uma superfície de, aproximadamente, $100 \mathrm{~km}^{2}$, alcança um comprimento máximo de $56 \mathrm{~km}$, uma largura máxima de cerca de $4 \mathrm{~km}$, e comporta um volume em torno de $530 \mathrm{Hm}^{3}$. A profundidade máxima da represa é de $18 \mathrm{~m}$ e a média de $5,2 \mathrm{~m}$.

Não houve desmatamento prévio, no reservatório; assim, a floresta inundada, morta, delimita os leitos originais dos rios que sofrem influência do represamento. Entre as árvores inundadas, macrófitas aquáticas tais como Eichhornia crassipes. Paspalum repens, Scirpus cubensis e Pistia stratiotes desenvolvem bem. (Junk, W. J., com. pess.).

Nos arredores da estação de coleta o leito original do rio mede cerca de $90 \mathrm{~m}$ de largura, e apresenta, em virtude da irregularidade do fundo, uma profundidade que varia entre $10 \mathrm{e}$ $15 \mathrm{~m}$. Registram-se na superfície, variações nos seguintes parâmetros: transparência da água (medida com Disco de Secchi) - 1,3 $4,5 \mathrm{~m}$; temperatura $-28,3-33,3^{\circ} \mathrm{C} ; \mathrm{pH}-$ $5,3-7,4$; condutividade elétrica $-22,24-$ $30,42 \mathrm{uS}_{20} / \mathrm{cm}$, e oxigênio dissolvido $-1,54$ $-5,55 \mathrm{mg} / 1$. (Darwich, A. J, com. pess.).

\section{MATERIAL E MÉTODOS}

No periodo de novembro 1977 a novembro 1978 , coletas de amostras quantitativas e qualitativas de Cladócera foram realizadas mensalmente na estação C-Una 45 , que se localiza no rio Curuá-Una, acima da desembocadura do rio Moju. (Fig. 2) . Refere-se a esta estação em função do número de quilômetros (medidos no leito original do rio) que a mesma dista da barragem, $\mathrm{Km} \mathrm{0}$. Para as coletas quantitativas utilizou-se uma bomba de água manual. Em cada metro de profundidade, 50 litros de água foram coletados e filtrados através de uma rede de plâncton de $55 \mu \mathrm{m}$. Para as coletas qualitativas, a rede de plâncton foi arrastada vertical e horizontalmente. Todas as coletas foram realizadas por volta das 11:00 horas. As amostras foram fixadas no campo com formol a $6-7 \%$. A contagem total dos cladóceros foi realizada com auxílio de uma lupa binocu-

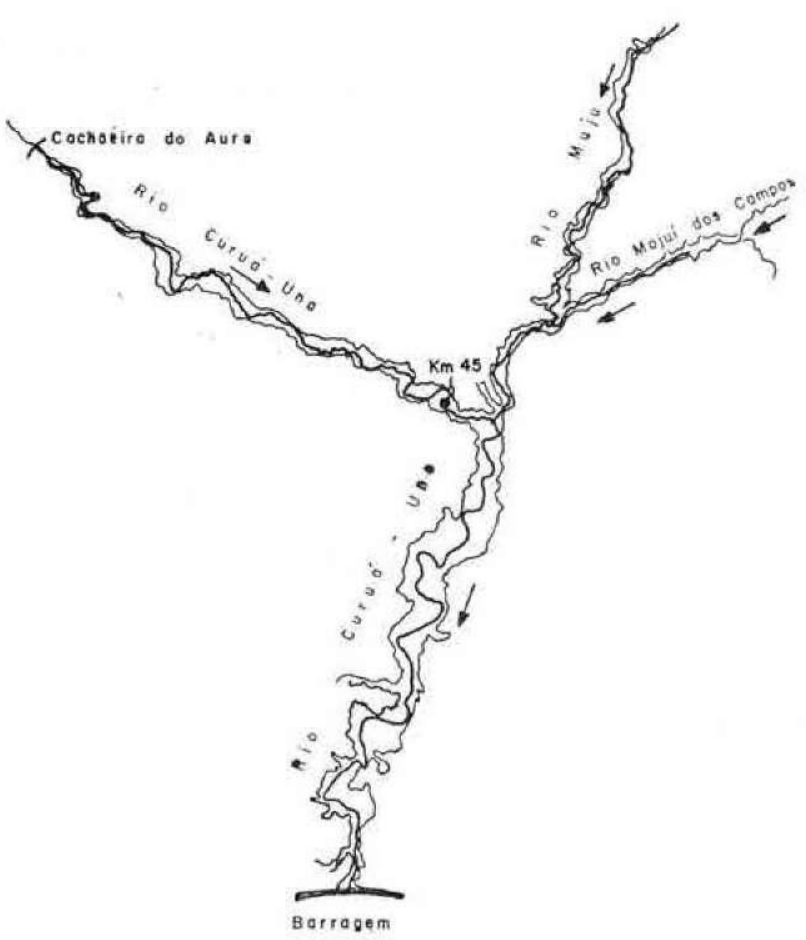

Fig. 2 - Levantamento topográfico da represa de CuruáUna na cota de $68 \mathrm{~m}$ (CELPA), e a localizaçăo da estação de coleta.

lar, Zeiss, de 10 a 40 vezes de aumento. Fê. meas partenogenéticas, fêmeas efipiais e machos foram contados juntos.

\section{REsultados}

A Fig. 3 mostra a abundância de $C$. cornuta e C. reticulata $\mathrm{em}$ cada mês de coleta. Com exceção de novembro 1977, ambas as espécies estiveram presentes durante todo período de estudo, se bem que, às vezes, com densidades muito baixas, como é o caso de $C$. reticulata no mês de novembro 1978. Observa-se pela Fig. 3 que enquanto $C$. reticulata apresenta dois máximos de abundância, um no período de janeiro e fevereiro 1978, e outro, de mais curta duração, no mês de outubro 1978, C. cornuta apresenta somente um máximo, prolonga. do, abrangendo os meses de julho a outubro 1978. Observa-se também que, de modo geral, a presença de fêmeas efipiais e machos das duas espécies coincide com os máximes da abundância .

Para apresentar a distribuição vertical de C. cornuta e C. reticulata (Fig. 4), o número total de cada espécie em cada profundidade 


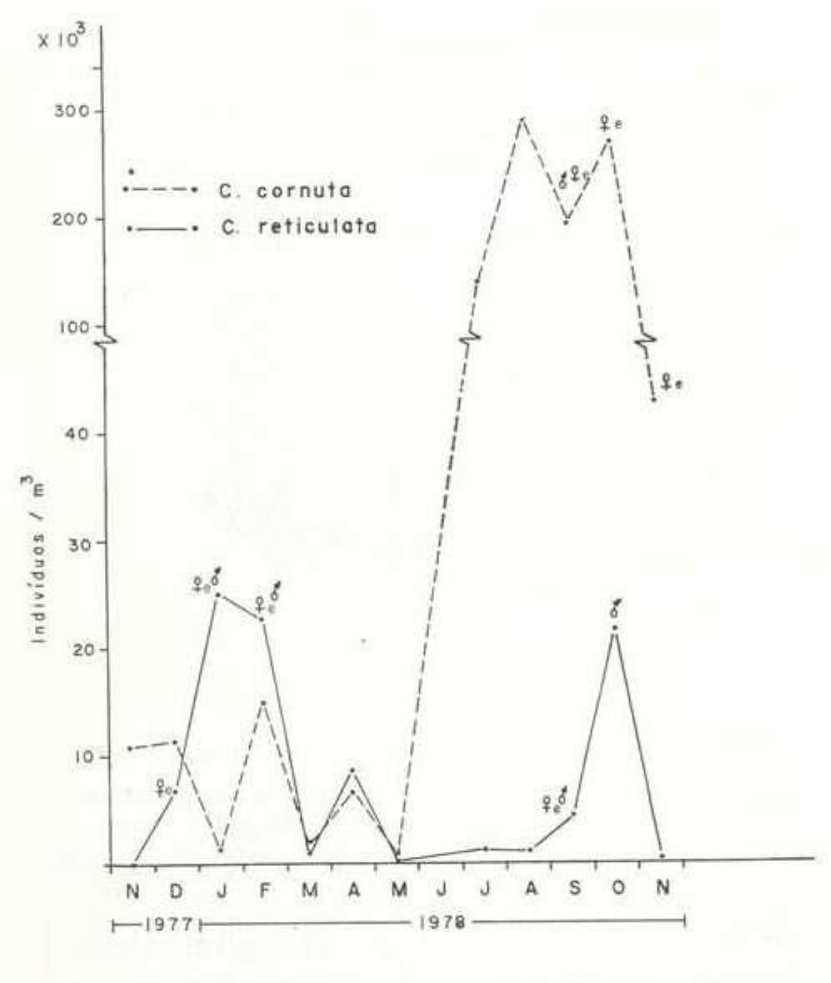

Fig. 3 - Densidade de C. cornuta e C. reticulata na Represa de Curuá-Una. (Presença de fêmeas efipiais e machos representados por $q$ e $\hat{\sigma}$ respectivamente).

coleteda foi convertido a uma percentagem do número total de Cladócera. Observa-se pela Fig. 4 que quando juntas na coluna d'água, as espécies apresentam uma estratificação vertical. C. cornuta ocorre com maior abundância nas camadas superficials, entre 0,0 e 4,0 metros, enquanto $C$. reticulata tende a concentrar-se abaixo de $C$. cornuta, nas camadas mais profundas da coluna d'água, inferiores a 5,0 metros.

A Fig. 5 mostra a faixa de tamanho encontrada para $C$. cornuta e $C$. reticulata provenientes da Represa de Curuá-Una. Este resultado foi obtido medindo 26 femeas partenogenéticas, ovadas, de cada espécie.

\section{DISCUSSÃO}

Zago (1976) comenta que a presença de duas ou mais espécies do gênero Ceriodaphnia não é incomum, e uma revisão da literatura mostra que diversos autores registram, entre outras espécies, a coexistência de $C$. cornuta e C. reticulata: Worthington \& Ricardo (1936) no lago Edward, África; Green (1965, 1971) nos lagos Mulehe e Albert, Uganda; Zago (1976) na Represa de Americana, São Paulo, Brandorff (1977) no lago Castanho, Amazonas, e Leal (com. pess.) no lago Manaquiri, Amazonas. De acordo com Pennak (1957), em situações de coexistência de espécies congenéricas, como é exemplificado acima, é de esperar-se que um ou mais mecanismos de segregação seja observado. No que se refere à presença de $C$. cornuta e $C$. reticulata na Represa de Curuá-Una, o presente estudo mostra evidências para três mecanismos de segregaçăo (separação no tempo, no espaço, e provavelmente em hábitos alimentares) atuarem, em conjunto, para permitir a coexistência destas congenéricas.

SEPARAÇÃo No TEMPo - Na Represa de Curuá-Una, as espécies $C$. cornuta e $C$. reticulata apresentam picos de abundância em épocas diferentes (Fig. 3), o que sugere que os animais estão segregados no tempo. Fenômeno semelhante foi encontrado por Brandorff (1977) no lago Castanho. Este autor observou dois máximos de abundância para cada espécie: para $C$. cornuta, um máximo coincidindo com um de C. reticulata, na época em que o nível d'água subia, e outro no período em que o nível d'água descia, e para $C$. reticulata, além do pico coincidente, um máximo na época em que o nível d'água se encontrava mais baixo.

Tanto no lago Castanho (Brandorff, 1977) quanto na Represa de Curuá-Una, o aparecimento de fêmeas efipiais e machos (formas mais raras do que as usuais fêmeas partenogenéticas) coincidem com as maiores densidades populacionais, o que nos leva a considerar a sugestão de Hutchinson (1967):

... diapausa, sem dúvida desenvolvido em sistemas de água doce principalmente para evitar períodos fisicamente desfavoráveis, pode também ser de importância prioritária para uma espécie ultrapassar situações biologicamente desfavoráveis que incluem os efeitos adversos de competição.

SEPARAÇÃo No ESPAÇO - Os resultados deste estudo mostram que, além de estarem separadas no tempo, quando juntas, $C$. cornuta e C. reticulata spresentam uma estratificação 

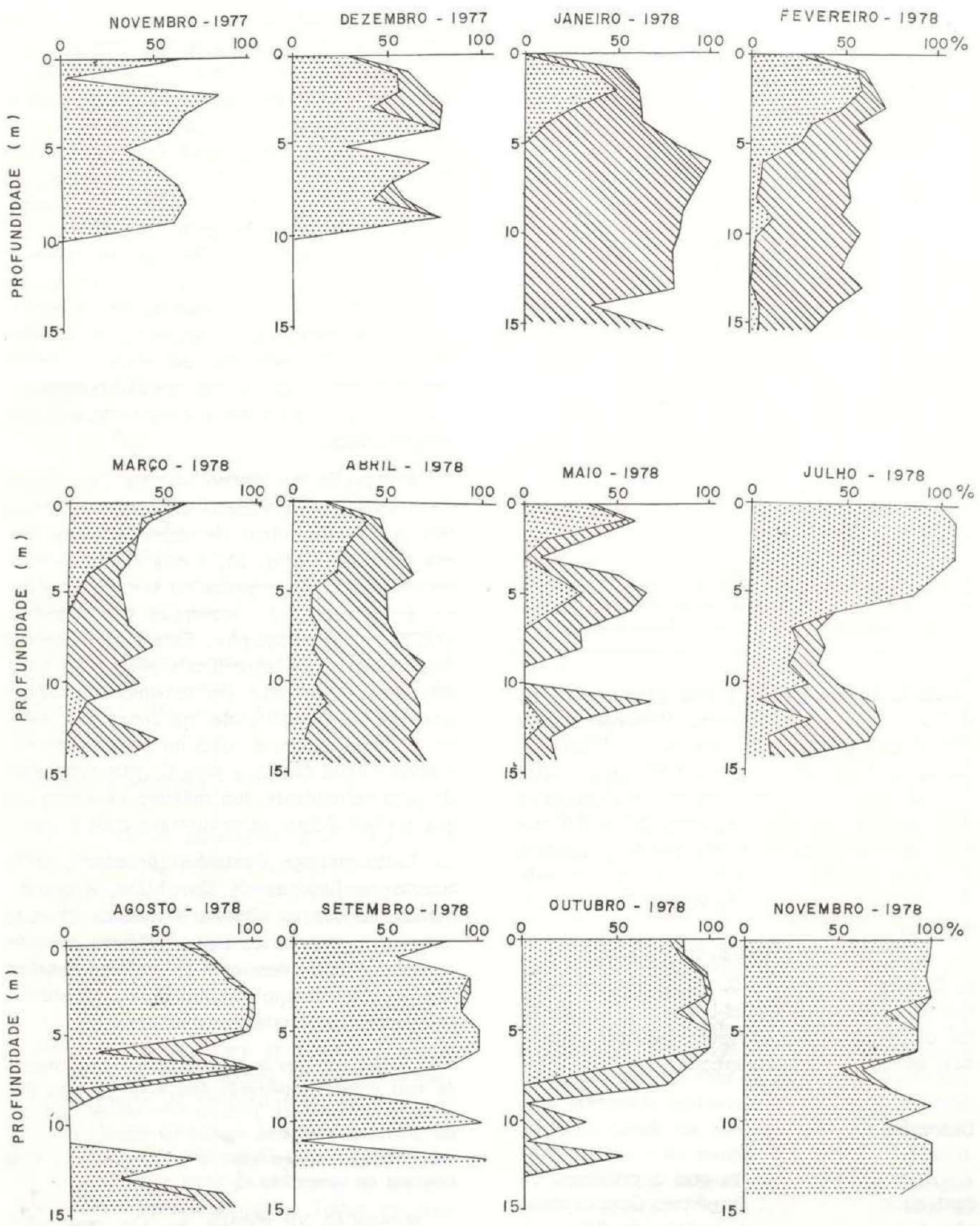

Fig. 4 - Distribuição vertical de Ceriodaphnia cornuta de Curuá-Una. 


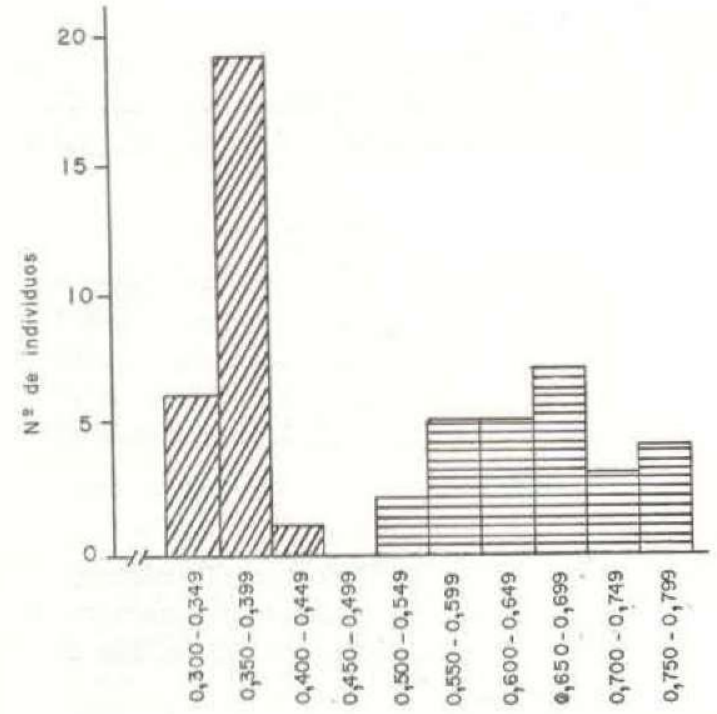

Fig. 5 - Intervalos de classes de comprimento (mm) de Ceriodaphnia cornuta $[I I I|l|$ e C. reticulata na Represa de Curuá-Una.

vertical. (Fig. 4). Este fenômeno aparentemente năo é restrito à Represa de Curuá-Una, pois, no lago Castanho, por exemplo, Brandorff (1977) encontrou jovens de C. cornuta concentradas nas camadas superficiais, entre 1 e $2 \mathrm{~m}$. e C. reticulata concentrada nas camadas mais profundas da coluna d'água, e algo semelhante foi observado por Worthington \& Ricardo (1936) no lago Edward. Neste lago, os autores identificaram três espécies de Ceriodaphnia, as quais, para fins de contagem, foram reunidas em dois grupos, um de formas maiores (C. reticulata e C. dubia), e outro de formas menores (C. cornuta e jovens das três espécies). Worthington \& Ricardo (1936) observaram que. enquanto a distribuição vertical dos dois grupos foi semelhante, a região de concentração das formas maiores foi um pouco mais profunda do que a das formas menores.

TAMANHO DAS ESPÉcies - Uma diferença no tamanho de espécies de crustáceos planctônicos implica em uma diferença em hábitos alimentares dado que o limite superior do tamanho da partícula filtrada é determinado pelo tamanho do indivíduo (Hutchinson, 1967; Sandercock, 1967; Bowers, 1980). Na realidade, este tenômeno tem sido mais bem constatado em copepodos; no entanto, no lago Albert, por exemplo, Green (1971) encontrando um comprimento entre $0,34-0,43 \mathrm{~mm}$ para $C$. cornuta e $0,47-0,64 \mathrm{~mm}$ para $C$. reticulata considera a diferença suficiente para as duas coexistirem. Os resultados obtidos neste estudo (Fig. 5) são semelhantes aos de Green (1971); assim, é provável que a diferença no tamanho das espécies congenéricas na Represa de Curuá-Una também seja suficiente para as duas coexistirem.

Em resumo, sugere-se que a presença de C. cornuta e $C$. reticulata na Represa de CuruáUna se deve à ação conjunta de três mecanismos de segregação. Sugere-se também que a coexistência, não tão rara, de $C$. cornuta e $C$. reticulata em lagos da Amazônia e possivelmente nos trópicos de modo geral, depende, assim como na Represa de Curuá-Una, de pelo menos um dos já bem estabelecidos mecanis. mos de segregação atuar, com outros fatores ainda não estudados, na separação destas espécies congenéricas.

\section{AGRADECIMENTOS}

Agradecemos ao Dr. W. J. Junk, E. R. Hardy, e Dr. V. E. Thatcher pela colaboração no sentido de sugestões e críticas, na elaboração deste trabalho.

\section{SUMMARY}

In a study conducted in the Curuá-Una Reservoir. Pará, there is evidence to suggest that three segregating mecanisms (temporal and spatial separation, and a sufficiente difference in size to infer in diet differences) operate together to permit the co-occurence of the congeneric species Ceriodaphnia cornuta and Ceriodaphnia reticulata.

\section{REFERENCIAS BIBLIOGRÁFICAS}

BOWERS, J.A.

1980 - Feeding habits of Diaptomus ashlandi and Diaptomus sicilis in Lake Michigan. Int. Revue. ges Hydrobiol., 65 (2): 259-267.

BRANDORFF, G-O.

1977 - Untersuchugen zur Populationsdynamik des Crustaceanplankton in Tropischen Lago Castanho (Amazonas, Brasilieien). Tese de Doutoramento. Universidade de Kiel. 108p.

GREEN, J.

1965 - Zooplankton of Lakes Mutanda, Bunyonyi and Mulehe. Proc. Zool. Soc. Lond., 144 (3): 383-402. 
1971 - Associations of Cladocera in the zooplankton of the lake sources of the Whifte Nile. J. of Zoology, 165 (3): 373-414.

HARDY, E.R.

1978 - Composição de Zooplâncton em Cinco Lagos da Amazônia Central. Tese de Mestrado, Dedto. Ciências Biológicas Univ. Fed. São Carlos. 143p.

HUTCHINSON, G.E.

1967 - A Treatise on Limnology V. 2 - Introduction to Lake Biology and the Limnoplankton. New York, John Wiley \& Sons. 1115p.

PENNAK, R.W.

1957 - Species composition of limnetic zooplankton communities. Limnol. \& Oceanogr., 2 (3): 222-232.

ROBERTSON, B.A.

1980 - Composição Abundância e Distribuição de Cladocera (Crustacea) na regiāo de água livre da Represa de Curuá-Una, Pará. Tese de Mestrado. FUA/INPA.CNPq. 105p.
SANDERCOCK, G.A.

1967 - A study of the selected mecanisms for the coexistence of Diaptomus spp. in Clarke Lake, Ontario. Limnol. \& Oceanogr., 12: 97112.

WORTHINGTON, E.B. \& RICARDO, C.K.

1936 - Scientific results fo the Cambridge Expedition to the Esst African Lakes 1930-1. N. 17. The vertical distribution and movements of the plankton in Lakes Rudolf, Naivasha, Edward, and Bunyonyi. J. Linn. Soc. Zoology, 40: $33-69$.

ZAGO, M.S.A.

1976 - The planktonic Cladocera (Crustacea) and aspects of eutrophication of Americana Reservoir, Brasil. Boln. Zool. Univ. Saao Paulo, 1: $105-145$.

(Aceito para publicação em 14/05/81) 